or treatment recommendations in specific subgroups. GDTs considered GLIA appraisal findings when they revised their reports and found the GLIA appraisals helpful in creating more implementable guidelines.

Implications for Guideline Developers/Users GLIA training for GDTs, and formal use of the GLIA tool help produce more implementable guidelines.

\section{PARTIALLY UPDATING A GUIDELINE TO IMPROVE ITS IMPLEMENTATION}

S Warttig, N Elliott. National Institute for Health and Clinical Excellence, Manchester, UK

\section{0:1136/bmjgs-2013-002293.93}

Background We were commissioned to partially update a clinical guideline. The remit was to develop new service delivery recommendations to support implementation of the guideline whilst leaving the existing clinical recommendations unchanged.

Objectives To describe the approach taken in partially updating a guideline to improve its implementation. To discuss the problems encountered and possible solutions.

Methods At the time, there was limited guidance on conducting service delivery evidence reviews. A methodology was developed and agreed by the developers, the NICE Methodology Team and the GDG which aimed to ensure the process was as robust, reproducible and transparent as possible.

Results Limited evidence was identified using the agreed methodology. This prevented identification of successful service delivery models. It also became apparent that that some of the implementation issues were embedded in the original guideline recommendations, and these could not be changed.

Discussion The methodology used could not adequately address the implementation issues, as it was not possible to amend any of the problematic recommendations, or describe a method of service delivery that was clinically and cost effective. Agreement could not be reached on how to progress with developing the recommendations, and so a decision was made to cease publication of the service delivery recommendations.

Implications for Guideline Developers/Users Partial updates are more challenging for guidelines requiring implementation support and should: 1) Go through a process to assess the issues before deciding how guideline should be updated. Or 2) Come with a remit to enable the developers to amend the recommendations for which implementation support is sought.

\section{ENHANCING THE UPTAKE OF CLINICAL PRACTICE GUIDELINES: THE DEVELOPMENT OF A GUIDELINE IMPLEMENTABILITY TOOL (GUIDE-IT)}

M Kastner, J Versloot, L Hayden, A Chatterjee, O Bhattacharyya. Li Ka Shing Knowledge Institute of St. Michael's Hospital, Toronto, Canada

\section{0:1136/bmjgs-2013-002293.94}

Background Guidelines have the potential to facilitate implementation of evidence into practice but this has not been consistently achieved. We developed a guideline implementability tool (GUIDE-IT), which can assess the implementability of guideline recommendations.

Objective To determine if GUIDE-IT can improve the Language and Format of guideline recommendations.
Methods Using a mixed-methods approach to develop GUIDEIT, we conducted 1) a Realist Review of guideline factors influencing uptake, and used its results to build a conceptual model of guideline implementability; 2) qualitative interviews with 20 family physicians to determine factors influencing guideline uptake and to obtain input on tool design; 3) created a prototype and conducted validity assessments with experts in guideline development and human factors. GUIDE-IT was then pilot tested with the Canadian Diabetes/Paediatric Associations (CDA, CPS) to determine its potential for assessing the implementability of guideline recommendations.

Results Pilot testing with CDA and CPS developers showed that factors across 4 sub-domains of Language (clarity, simplicity, specificity, and actionability) and 3 sub-domains of Format (presentation, components, and multiple versions) were applicable for modifying recommendations. GUIDE-IT was feasible to use by guideline developers to identify implementability problems and to improve recommendations.

Discussion GUIDE-IT is based on a robust evidentiary base with the potential to improve guidelines. Next steps include evaluating GUIDE-IT in a controlled trial to determine its impact on end-user clinical decision making.

Implications for Guideline Developers/Users GUIDE-IT has potential to be a practical tool for developers to improve the language and format of guideline recommendations.

\section{IDENTIFYING, DESCRIBING AND EVALUATING GUIDELINE IMPLEMENTABILITY TOOLS}

${ }^{1} \mathrm{~J}$ Cheng, ${ }^{1} \mathrm{~A}$ Gagliardi, ${ }^{2} \mathrm{M}$ Brouwers, ${ }^{3} \mathrm{O}$ Bhattacharyya. ${ }^{1}$ University Health Ne; ${ }^{2}$ Department of Oncology, McMaster University, Hamilton, Canada; ${ }^{3}$ St. Michael's Hospital, Toronto, Canada

\section{0:1136/bmjqs-2013-002293.95}

Background Research shows that guidelines are more easily translated to practice when accompanied by information that helps users accommodate, implement and evaluate use of the recommendations. Guidelines vary in whether and how they offer such information, which we refer to as guideline implementability tools (GItools).

Objectives To identify, describe and evaluate exemplar GItools that address Resource Implications, Implementation and Evaluation, and suggest how they could be improved.

Methods GItools were identified in several sources (guidelines, Medline, professional organisation web sites, Implementation Science, Internet, expert referrals) and two individuals independently assessed each on criteria recommended by G-I-N members: purpose statement, instructions for use, citations for source of content, and how it was developed.

Results The search produced 228 potential tools. Of these 94 were ineligible and 63 met no assessment criteria. Of the remaining 71 tools, 13 (18.3\%), 24 (33.8\%), 23 (32.4\%) and 11 (15.5\%) met 1, 2, 3 and 4 criteria, respectively; and 57 (80.3\%), $37(52.1 \%), 41(57.7 \%)$ and $41(57.7 \%)$ provided purpose, instructions, citations and development details, respectively. Most tools addressed Implementation (44, 62.0\%). Twenty-eight (39.4\%) were guideline-specific and $43(60.6 \%)$ were generic.

Discussion Few GItools met all assessment criteria. GItools could be more informative across all criteria. Few GItools were available to help users assess resource needs or evaluate guideline use. Many GItools were applicable to a variety of guidelines.

Implications We identified a number of ways to improve GItools. Collaborative development and sharing of both generic 